\title{
Hydrogel scaffolds composed of genetically synthesized self-assembling peptides for three-dimensional cell culture
}

\author{
Masayasu Mie, Mayu Oomuro and Eiry Kobatake \\ Peptides were genetically produced that were composed of two or three repeats of the self-assembling peptide RADA16 \\ (RADARADARADARADA), and referred to as RADA16 $\times 2$ and RADA16 $\times 3$, respectively. These peptides were expressed as \\ fusion proteins that retained the activity of the fusion partner protein. The expressed peptides exhibited both fibril formation \\ and the ability to support cell adhesive activity. Moreover, hydrogels formed by the peptides via the addition of a medium \\ provided a three-dimensional environment for cell proliferation.
}

Polymer Journal (2013) 45, 504-508; doi:10.1038/pj.2012.216; published online 12 December 2012

Keywords: 3-D cell culture; genetically synthesis; hydrogel; self-assembling peptide

\section{INTRODUCTION}

The development of biomaterials that support cellular functions, such as cell adhesion, growth and differentiation, is important for tissue engineering. ${ }^{1}$ In an effort to create such novel biomaterials, artificial extracellular matrices (ECMs) have been developed by various groups. $^{2-5}$

Artificial ECM proteins have been designed and genetically synthesized in our laboratory. ${ }^{6-10}$ Our strategy for the design of artificial ECM proteins relies on the combination of structural peptides and active functional peptides that control cellular functions. One of the ECM proteins previously constructed in our lab, named ERE, is composed of 12 repeats of the elastin-derived APGVGV peptide motif as the stable structural peptide, with the cell adhesive RGD peptide motif as the active functional peptide. ${ }^{6,7}$

ERE can be immobilized on a hydrophobic surface via the hydrophobic APGVGV peptide motif and exhibits cell adhesive activities as a result of the RGD peptide. Multi-functional ECM proteins have also been constructed based on the ERE protein. EREI, which consists of ERE fused with the IKVAV peptide, not only exhibits cell adhesive activity but also promotes angiogenesis and neural differentiation as a result of the combination of IKVAV and RGD. ${ }^{8-12}$ In our genetic engineering-based designs, multi-functional ECMs composed not only of peptide motifs but also of growth factors were constructed. Epidermal growth factor fused to the ERE protein was found to exhibit cell growth activity via the epidermal growth factor moiety. ${ }^{7}$ Our genetically designed ECM proteins based on ERE demonstrated applicability as biomaterials. ${ }^{6-10}$ However, covalent cross-linking is required to form a hydrogel structure in order to apply our designed ECM proteins to three-dimensional (3-D) culture. ${ }^{13}$ The use of cross-linking reagents can result in the loss of the functional peptide's activity.

To overcome this problem, we focused on self-assembling peptides. One such self-assembling peptide, named RADA16 (RADARADARADARADA), is a designed oligopeptide whose sequence is based on EAK16 (AEAEAKAKAEAEAKAK), which was originally identified as a set of tandem repeats in the yeast protein Zuotin. ${ }^{14,15}$ To design RADA16, the lysine and glutamic acid residues of EAK16 were altered to arginine and aspartic acid residues, respectively, and the sequence order was modulated. The resulting RADA16 peptide is composed of four repeating units of the RADA amino-acid sequence and forms a stable $\beta$-sheet structure. RADA16 spontaneously assembles in the presence of monovalent salt or under physiological conditions as a result of both the ionic side chain interactions and the conventional $\beta$-sheet propensity of the backbone, ultimately forming a macroscopic hydrogel known to facilitate cell culture. ${ }^{14,16-18}$

Cell attachment and neurite outgrowth were enhanced when neural cells were cultured on this hydrogel. Moreover, cells may be embedded in RADA16 peptide hydrogels, providing a 3-D environment for the cells to grow. Various functional units were also incorporated into the RADA16 peptide in these 3-D cultures. For example, a peptide hydrogel functionalized with bone marrow homing peptide in addition to RADA16 not only enhanced cell attachment but also promoted neural stem cell differentiation without the addition of soluble growth factors. ${ }^{17}$ These experiments demonstrate the applicability of attaching a functional peptide motif to RADA16. In these experiments, however, the functionalized self-assembling 
peptides were chemically synthesized, presenting a limitation for functional motifs that can fuse to RADA16. For example, it is difficult to add functional motifs via chemical synthesis that have large molecular weights, such as growth factors. Therefore, genetic engineering methods may provide an opportunity for the construction of self-assembling peptides to be used in multifunctional hydrogel scaffolds.

In this study, we have genetically produced RADA16 peptides. Double or triple repeats of RADA16, named RADA16 $\times 2$ or RADA $16 \times 3$, were fused for intein-mediated purification with affinity chitin-binding tag and expressed in E. coli. The peptides were purified by the intein-mediated purification with affinity chitin-binding tag system, which is based on intein-specific self-cleavage, and subsequent fibril formation was confirmed. Finally, cells were cultured in the RADA16 $\times 3$ peptide hydrogel scaffold.

\section{MATERIALS AND METHODS}

\section{Plasmid construction}

For construction of pET32c-RADA16, point mutations were introduced into pET32c (Merck KGaA, Darmstadt, Germany) for removal of BssHII recognition sequences by point-mutagenesis using primer sets $5^{\prime}$-GATGGCGCTGGG CGCAATGCGTGCCATTACCGAGTCCGGGCT- $3^{\prime}$ and $5^{\prime}$-AGCCCGGACTCG GTAATGGCACGCATTGCGCCCAGCGCCATC- $3^{\prime}$. The resulting plasmid, pET32c-BssHII mut, was digested with $\mathrm{NcoI}$ and EcoRI. The synthesized a

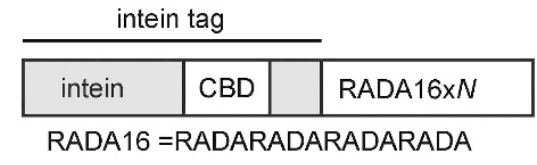

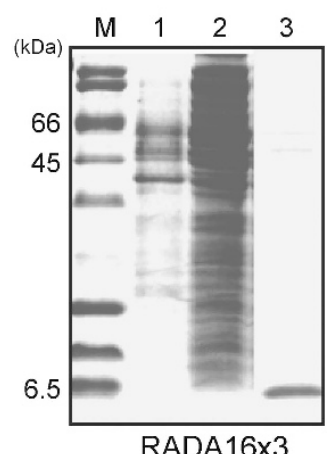

RADA16x2 oligonucleotides, 5'-CATGGCCCGTGCTGACGCTCGTGCTGACGCTCGTGC TGACGCTCGTGCTGACGCGCGCTAAG- $3^{\prime}$ and $5^{\prime}$-AATTCTTAGCGCGCGT CAGCACGAGCGTCAGCACGAGCGTCAGCACGAGCGTCAGCACGGGC-3', were mixed, heated and cooled to form double-strand DNA. The annealed oligonucleotide, NcoI-RAD16-I-BssHII-EcoRI, was inserted into digested pET32c-BssHII. The resulting plasmid was termed pET32c-RADA16. After confirming the sequence of pET32c-RADA16, annealed synthetic oligonucleotides (5'-CGCGTGCTGACGCTCGTGCTGACGCTCGTGCTGACGCTCGTGC TGACGCGCGCTAAG- $3^{\prime}$ and $5^{\prime}$-AATTCTTAGCGCGCGTCAGCACGAGCGT CAGCACGAGCGTCAGCACGAGCGTCAGCA-3'), BssHII-RADA16-I-BssHIIEcoRI, were inserted into pET32c-RADA16 digested with BssHII and EcoRI to produce pET32c-RADA $16 \times 2$. Plasmid $\mathrm{pET} 32 \mathrm{c}-\mathrm{RADA} 16 \times 3$ was constructed using the same procedure. The RADA16 $\times 2$ or RADA16 $\times 3$ sequences were then amplified by PCR using primer sets ( $5^{\prime}$-GGTTGCTCTTCCAACCGTGCT

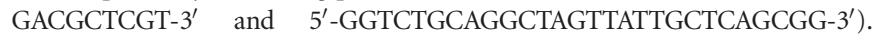
Amplified fragments were cloned into pUC18, and their sequences were confirmed. The resulting plasmids, pUC18-SapI-RADA16 $\times 2-P s t \mathrm{I}$ and pUC18-SapI-RADA16 $\times 3$-PstI, were digested with SapI and PstI. The digested fragments were inserted into pTYB21 (New England BioLabs, Ipswich, MA, USA) that was digested with the same restriction enzymes. The resulting plasmids were termed pTYB21-RADA16 $\times 2$ and pTYB21-RADA16 $\times 3$, respectively.

\section{Expression and purification of constructed proteins}

Constructed plasmids were transformed into E. coli BL21(DE3) competent cells (Merck KGaA) by heat shock. Transformed cells were cultured in Lysogeny Broth medium with ampicillin at $37^{\circ} \mathrm{C}$. Protein expression was induced by the addition of isopropyl- $\beta$-D-thiogalactopyranoside at a final concentration of $1 \mathrm{~mm}$. After incubation at $30^{\circ} \mathrm{C}$ for $16 \mathrm{~h}$, cells were pelleted, resuspended in cell lysis buffer (20 mm Tris- $\mathrm{HCl}, 500 \mathrm{~mm} \mathrm{NaCl}, 1 \mathrm{~mm}$ EDTA, pH 8.5) and sonicated. After centrifugation, supernatants were applied to chitin beads (New England BioLabs) equilibrated with column buffer (20 mm Tris-HCl, $500 \mathrm{~mm} \mathrm{NaCl}, \mathrm{pH} 8.5$ ), incubated for $60 \mathrm{~min}$ at $4{ }^{\circ} \mathrm{C}$, and washed with column buffer. Then, to release the target protein, on-column cleavage was induced by the addition of cleavage buffer $(20 \mathrm{~mm}$ Tris- $\mathrm{HCl}, 500 \mathrm{~mm} \mathrm{NaCl}, 50 \mathrm{~mm}$ dithiothreitol, $1 \mathrm{~mm}$ EDTA, pH 8.5) and incubated for $40 \mathrm{~h}$ at $25^{\circ} \mathrm{C}$. The released proteins were dialyzed against ultrapure water. Purified proteins were analyzed by Tricin SDS-polyacrylamide gel electrophoresis (17.5\% acrylamide gels), and the concentrations of purified proteins were measured with a BCA protein assay reagent kit (Thermo Scientific, Rockford, IL, USA).

\section{Congo-red-binding assay}

Congo red was dissolved in buffer consisting of $150 \mathrm{mM} \mathrm{NaCl}, 16 \mathrm{~mm}$ $\mathrm{Na}_{2} \mathrm{HPO}_{4}$ and $4 \mathrm{~mm} \mathrm{NaH} \mathrm{NO}_{4}$ adjusted to $\mathrm{pH}$ 7.4. This solution was added to various concentrations of proteins resulting in final protein concentrations of $6.25,12.5,25,50$ and $100 \mu \mathrm{m}$. After incubation for $30 \mathrm{~min}$ at room temperature, absorbance between 350 and $700 \mathrm{~nm}$ was measured by spectrophotometer.

\section{Transmission electron microscopic observation}

Various concentrations $(6.25,12.5,25,50,100 \mu \mathrm{M})$ of purified proteins in phosphate-buffered saline were incubated at room temperature for $1 \mathrm{~h}$ and fraction, 2 , soluble fraction, 3 , eluted peptide.
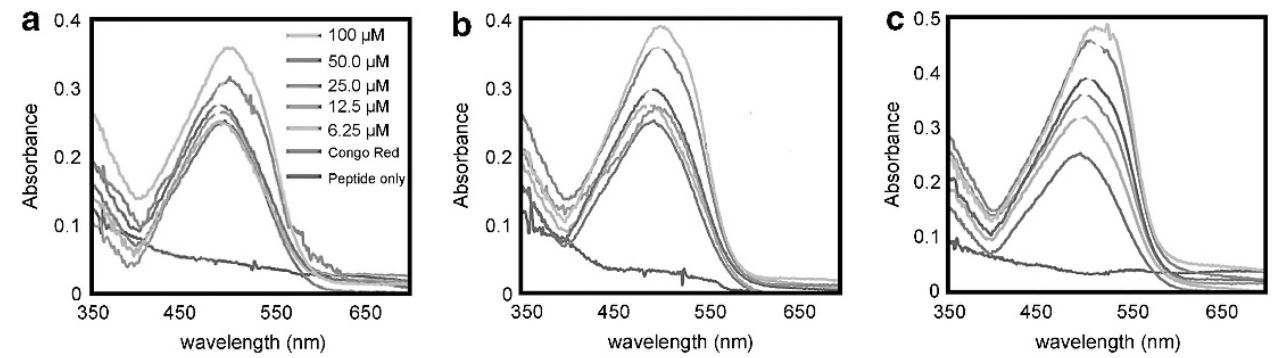

Figure 2 Congo-red-binding assay. (a) RADA16, (b) RADA16 $\times 2$, (c) RADA16 $\times 3$. Peptide concentrations were 6.25, 12.5, 25.0, 50.0, 100 $\mu$ M. A full color version of this figure is available at Polymer Journal online. 
adsorbed for $1 \mathrm{~min}$ on a specimen grid covered with a collodion membrane that was hydrophilically treated by ion coating. After washing with ultrapure water, samples were stained with $0.25 \%$ phosphotungstic acid for $1 \mathrm{~min}$ and dried on filter paper at room temperature for more than $2 \mathrm{~h}$. All images were obtained using an H-7500 electron microscope (Hitachi, Tokyo, Japan) at $100 \mathrm{kV}$.

\section{Cell culture}

The human lung adenocarcinoma epithelial cell line, A549 (RCB0098), was provided by the RIKEN BioResource Center (RIKEN BRC, Ibaraki, Japan). Cells were grown in Dulbecco's modified Eagle's medium (DMEM) with $10 \%$ fetal bovine serum and antibiotics $\left(100 \mathrm{U} \mathrm{ml}^{-1}\right.$ penicillin, $100 \mu \mathrm{g} \mathrm{ml}^{-1}$ streptomycin) at $37^{\circ} \mathrm{C}$ under $5 \% \mathrm{CO}_{2}$.

\section{Evaluation of cell adhesive activity}

Purified peptides $(50 \mu \mathrm{M})$ were added to non-tissue-culture 24-well plates (SUMILON MS-8024R, Sumitomo Bakelite, Tokyo, Japan) and incubated for $16 \mathrm{~h}$ at $37^{\circ} \mathrm{C}$. After removal of the peptide solution, cells were suspended in serum-free DMEM and seeded at $5.0 \times 10^{4}$ cells per well. After incubation for 2, 6 or $24 \mathrm{~h}$, the cells were washed to remove non-attached cells and then observed by microscopy. For evaluation of cell adhesive activity, attached cells in three randomly chosen fields of a dish were counted. Each assay was repeated three times.

\section{Cell culture in peptide hydrogel scaffold}

Purified peptides were dissolved in $10 \%(\mathrm{w} / \mathrm{v})$ sucrose at a concentration of $1 \mathrm{~mm}$. Five-hundred microliters of peptide solution was mixed with the same volume of cells $\left(2.0 \times 10^{4}\right.$ cells per $\left.\mathrm{ml}\right)$ in $10 \%(\mathrm{w} / \mathrm{v})$ sucrose. Fifty microliters of this mixture was placed in 96-well plates (Coaster 3595, Sigma-Aldrich, St Louis, MO, USA). After addition of serum-free DMEM (150 $\mu$ l per well), droplets were incubated at $37^{\circ} \mathrm{C}$ for $30 \mathrm{~min}$ to form a hydrogel. The cellcontaining hydrogels were then washed twice with DMEM containing 10\% fetal bovine serum. The cells were cultured at $37^{\circ} \mathrm{C}$ under $5 \% \mathrm{CO}_{2}$. The medium was changed every 2 days. After a culture period (1, 3, 5 and 7 days), $50 \mu \mathrm{l}$ of calcein AM (Dojindo, Kumamoto, Japan) dissolved in phosphatebuffered saline at a concentration of $10 \mu \mathrm{M}$ was added to cell-containing hydrogels to stain for living cells. After a 30-min incubation with calcein AM, the cells in the hydrogel were observed by using confocal laser scanning microscopy (FV300, Olympus, Tokyo, Japan).

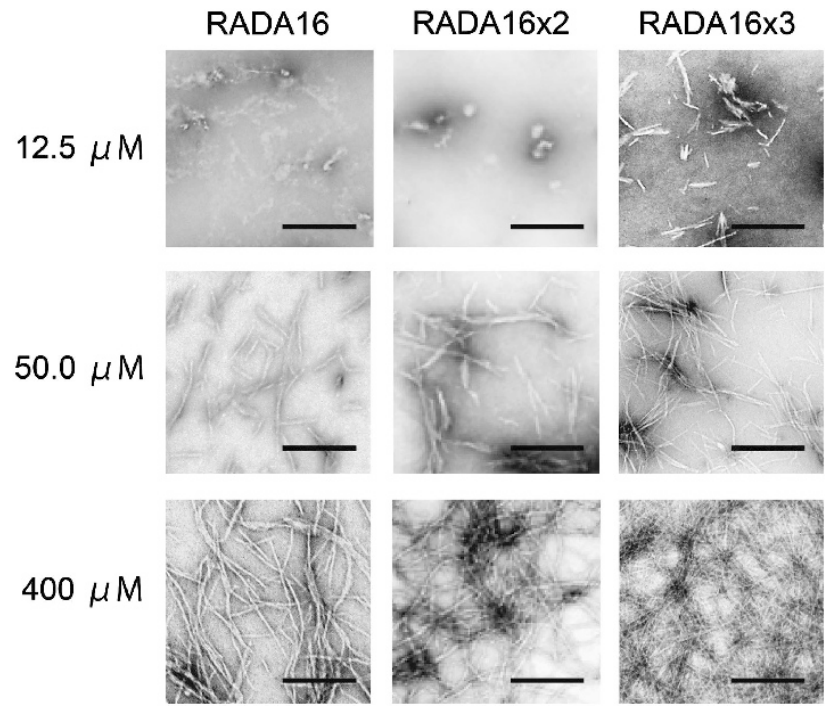

Figure 3 Transmission electron microscope images of the fibril formation of peptides. All scale bars represent $250 \mathrm{~nm}$.

\section{RESULTS AND DISCUSSION}

Expression and purification of genetically engineered RADA16 RADA16, composed of four repeating units of the RADA amino-acid sequence, is a well-studied self-assembling peptide. RADA16 is typically synthesized chemically and is commercially available as PuraMatrix (BD, Franklin Lakes, NJ, USA). In this study, double and triple repeats of RADA16, named RADA16 $\times 2$ and RADA16 $\times 3$, were genetically synthesized.

The construction of the RADA16 peptides is shown in Figure la. For purification by intein-mediated purification using an affinity chitin-binding tag system, RADA16xN (where $N=2$ or 3 ) was fused with the intein and the chitin-binding domain. The fusion proteins were expressed in E. coli BL21(DE3) and purified from the resulting soluble fraction, and the expressed proteins were added to the chitin column. After washing, dithiothreitol solution was added to the column for the induction of intein-specific self-cleavage, which releases the RADA16 $\mathrm{xN}$ proteins. The released RADA16 $\mathrm{xN}$ proteins

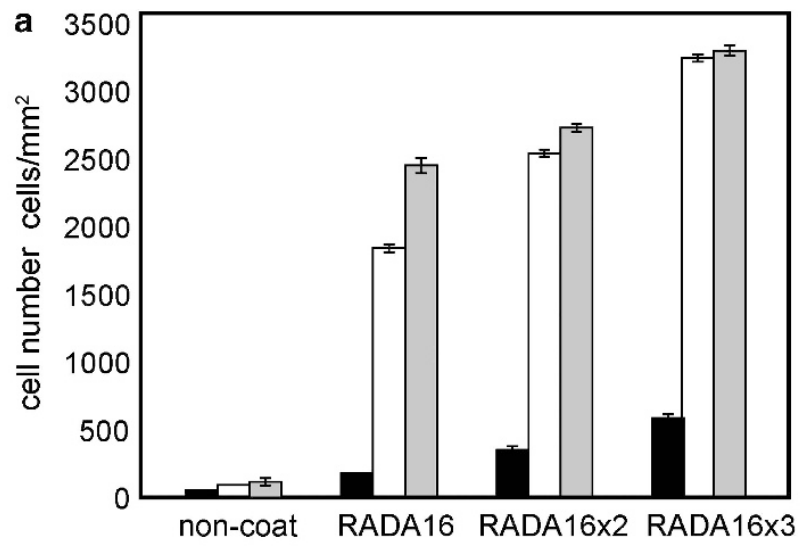

b
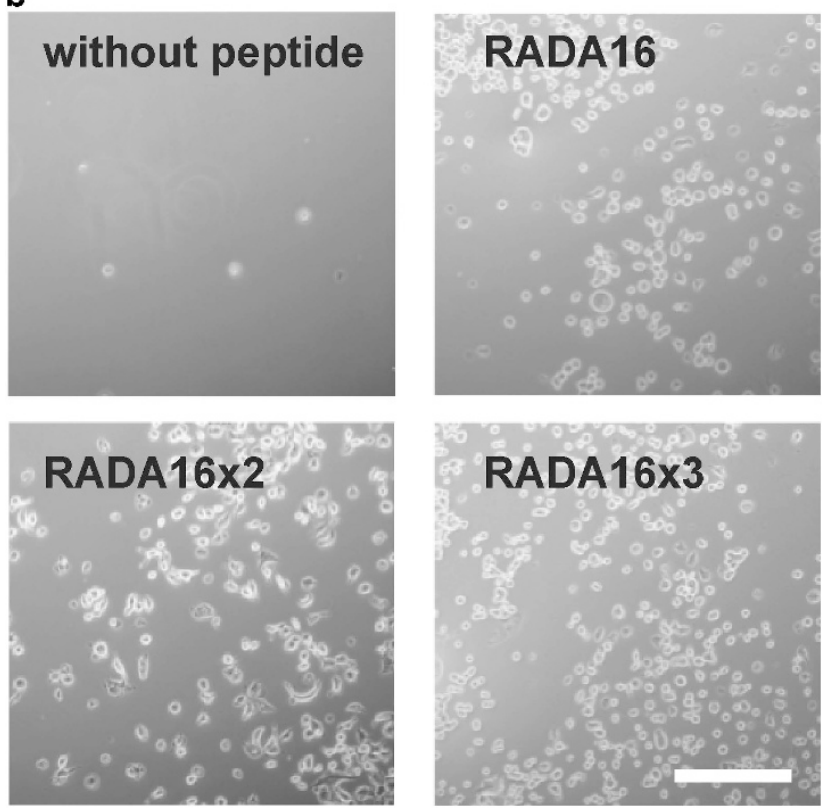

Figure 4 Evaluation of cell adhesive activity. (a) Number of attached cells on peptide-coated plates. Cells were suspended in serum-free Dulbecco's modified Eagle's medium and incubated for $2 \mathrm{~h}$ (black bar), $6 \mathrm{~h}$ (white bar) or $24 \mathrm{~h}$ (gray bar). (b) Images of attached cells on peptide-coated plates for $24 \mathrm{~h}$. Scale bar represents $100 \mu \mathrm{m}$. A full color version of this figure is available at Polymer Journal online. 
were dialyzed against ultrapure water. The purified proteins were analyzed by SDS-polyacrylamide gel electrophoresis (Figure 1b), and both peptides appeared in their expected size ranges when compared with a molecular size standard.

RADA16xN peptides were fused with intein and chitin-binding domains and subsequently expressed in E. coli. Moreover, the intein fragment was shown to exhibit its enzymatic activity even when fused to RADA16xN. Therefore, RADA16xN could be expressed as a fusion protein with a functional protein, such as a growth factor.

Evaluation of $\beta$-sheet formation by Congo-red-binding assay RADA16 is known to form a $\beta$-sheet structure. To confirm the formation of $\beta$-sheets by RADA $16 \times 2$ and $\operatorname{RADA} 16 \times 3$, peptide solutions were mixed with phosphate-buffered saline-containing Congo red. Congo red is a well-known amyloidophilic dye that exhibits a redshift in its absorbance maximum upon binding to amyloid fibrils. ${ }^{19,20}$ In this study, the $\beta$-sheet formation of expressed RADA16xN was evaluated and compared with that of RADA16 (Figure 2). In the case of RADA16, the redshift began at $50 \mu \mathrm{M}$ and became larger with increasing peptide concentrations. This result suggests that fibril formation depends on the number of RADA repeats in solution. Genetically produced RADA16 $\times 2$ showed similar results to RADA16 even though the number of RADA sequences was twice as high at the same concentration. However, RADA16 $\times 3$ exhibited a redshift in the absorbance maximum at a lower peptide concentration $(12.5 \mu \mathrm{M})$ than that of either RADA16 or RADA16 $\times 2$. Taken together, genetically engineered RADA16 peptides can spontaneously form $\beta$-sheet structures in the presence of sodium. Moreover, it is suggested that $\beta$-sheet structure formation can be enabled at lower peptide concentrations by increasing the number of repeating units of the RADA sequence.

Fibril observation by transmission electron microscope

After confirming $\beta$-sheet formation via the Congo-red-binding assay, fibril formation was observed by transmission electron microscope (Figure 3). At lower concentrations, all peptides $($ RADA16, RADA16 $\times 2$ and RADA16 $\times 3$ ) formed aggregates rather than fibrils. However, at higher peptide concentrations (RADA16 and RADA16 $\times 2>50 \mu \mathrm{M}, \quad$ RADA1 $6 \times 3>12.5 \mu \mathrm{M})$, all peptides were found to exhibit fibril formation. This result is consistent with the results of the Congo red assay, as the redshifts of Congo red began at almost identical concentrations to those required for fibril formation. Differences in the shapes of the fibrils were not observed. The diameters of all fibrils were $\sim 8 \mathrm{~nm}$, in accordance with previous literature. ${ }^{14}$ However, the fibril densities of each protein at a concentration of $400 \mu \mathrm{m}$ were different. The fibril densities increased with an increasing number of RADA repeating units. RADA16 $\times 3$ exhibited the highest fibril density. Therefore, increasing the number of RADA16 repeats has a similar effect as that of increasing the concentration of RADA16.

\section{Cell adhesive activity of RADA16xN}

RADA16 is known to exhibit cell adhesive activity. To confirm the cell adhesive activity of both RADA16 $\times 2$ and RADA16 $\times 3$, A549 cells were seeded onto RADA16 $\mathrm{xN}$-coated dishes (Figure 4). Non-tissueculture dishes were coated with RADA16xN peptides before cell seeding. Peptides were attached to the dish surface via hydrophobic interactions. For cell seeding, cells were suspended in serum-free DMEM to eliminate the effects of serum. Cells were not found to be attached on the non-coated dishes, even after $24 \mathrm{~h}$ of incubation. However, on the peptide-coated dishes, the number of attached cells increased with increasing incubation time. These results demonstrate that the RADA sequences retained cell adhesive activity even after increasing the number of repeating units and that cell adhesive activity increased as a result of increased fibril formation, which was dependent on the number of RADA repeating units.

Cell culture in the peptide hydrogel scaffold

Finally, cells were cultured in the RADA16 $\times 3$-derived hydrogels. Cells were inoculated in the hydrogel by mixing, and the cells in the

a
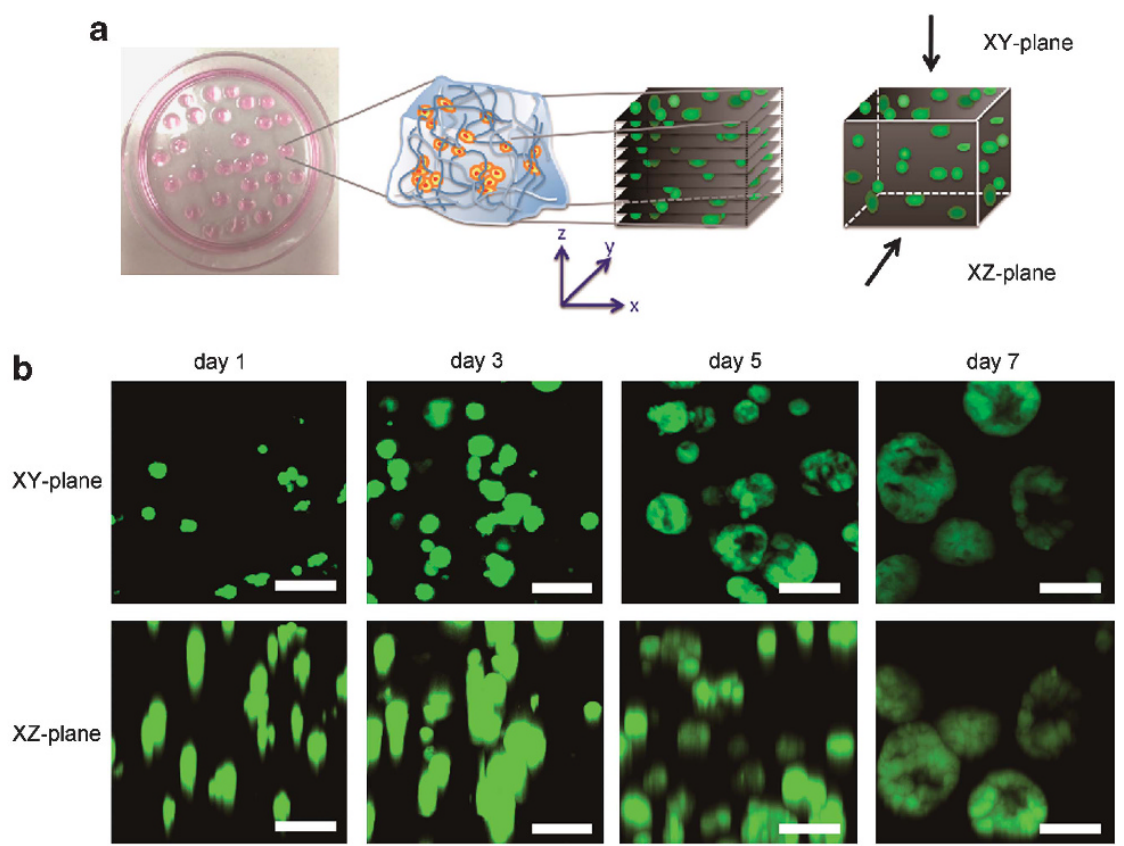

Figure 5 Cells grown in RADA $16 \times 3$ hydrogel. (a) Schematic drawings of cells observed in hydrogel. Cells cultured in hydrogel were stained with calcein AM and observed by confocal laser scanning microscopy. (b) Images of confocal laser scanning microscopy. All scale bars represent $100 \mu \mathrm{m}$. 
hydrogel were observed by confocal laser scanning microscopy (Figure 5a). In the dish culture, cells were attached on the plate surface and spread (data not shown). In contrast, in the hydrogel, culture cells were shown to form spheres and grow three-dimensionally (Figure 5b). After 7 days, the diameter of the spheres was $\sim 100 \mu \mathrm{m}, \sim 10$ times larger than those formed after 1 day. This result demonstrates that the cells were able to both survive and grow in the RADA $16 \times 3$ hydrogel matrix. Taken together, these results suggest that RADA $16 \times 3$ can form a hydrogel scaffold suitable for threedimensional cell culture.

\section{CONCLUSIONS}

The self-assembling peptide RADA16 was genetically expressed as a fusion protein that retained the activity of the fusion protein partner. The double and triple repeats of RADA16 expressed in E. coli retained both their fibril formation and cell adhesive abilities. Cells were able to both survive and grow in the RADA $16 \times 3$ hydrogel. Our results demonstrate that RADA16 can be genetically produced and applied to the construction of self-assembling, peptide-based, multi-functional scaffolding.

\section{ACKNOWLEDGEMENTS}

We are grateful to Ms Keiko Yamamichi from the Technical Department of the Tokyo Institute of Technology for technical support with the transmission electron microscope imaging.

1 Langer, R. \& Vacanti, J. P. Tissue engineering. Science 260, 920-926 (1993).

2 Barrera, D. A., Zylstra, E., Lansbury, P. T. \& Langer, R. Synthesis and RGD peptide modification of a new biodegradable copolymer -poly (lactic acid-co-lysine). J. Am. Chem. Soc. 115, 11010-11011 (1993)

3 Betre, H., Setton, L. A., Meyer, D. E. \& Chilkoti, A. Characterization of a genetically engineered elastin-like polypeptide for cartilaginous tissue repair. Biomacromolecules 3, 910-916 (2002)

4 Halstenberg, S., Panitch, A., Rizzi, S., Hall, H. \& Hubbell, J. A. Biologically engineered protein-graft-poly(ethylene glycol) hydrogels: a cell adhesive and plasm in-degradable biosynthetic material for tissue repair. Biomacromolecules 3, 710-723 (2002).
5 Nagaoka, M., Jiang, H. L., Hoshiba, T., Akaike, T. \& Cho, C. S. Application of recombinant fusion proteins for tissue engineering. Ann. Biomed. Eng. 38, 683-693 (2010).

6 Kobatake, E., Onoda, K., Yanagida, Y. \& Aizawa, M. Design and gene engineering synthesis of an extremely thermostable protein with biological activity. Biomacromolecules 1, 382-386 (2000).

7 Elloumi, I., Kobayashi, R., Funabashi, H., Mie, M. \& Kobatake, E. Construction of epidermal growth factor fusion protein with cell adhesive activity. Biomaterials 27, 3451-3458 (2006)

8 Nakamura, M., Mie, M., Mihara, H. \& Kobatake, E. Construction of multi-functional extracellular matrix proteins that promote tube formation of endothelial cells. Biomaterials 29, 2977-2986 (2008).

9 Nakamura, M., Mie, M., Mihara, H. \& Kobatake, E. Construction of a multi-functional extracellular matrix protein that increases number of N1E-115 neuroblast cells having neurites. J. Biomed. Mater. Res. B Appl. Biomater. 91, 425-432 (2009).

10 Nakamura, M., Yamaguchi, K., Mie, M., Akita, K. \& Kobatake, E. Promotion of angiogenesis by an artificial extracellular matrix protein containing the laminin-1 derived IKVAV sequence. Bioconjug. Chem. 20, 1759-1764 (2009).

11 Grant, D. S., Kinsella, J. L., Fridman, R., Auerbach, R., Piasecki, B. A., Yamada, Y., Zain, M. \& Kleinman, H. K. Interaction of endothelial-cells with a laminin-a chain peptide (SIKVAV) in vitro and induction of angiogenic behavior in vivo. J. Cell. Physiol. 153, 614-625 (1992).

12 Tashiro, K., Sephel, G. C., Weeks, B., Sasaki, M., Martin, G. R., Kleinman, H. K. \& Yamada, Y. A synthetic peptide containing the IKVAV sequence from the a-chain of laminin mediates cell attachment, migration, and neurite outgrowth. J. Biol. Chem. 264, 16174-16182 (1989)

13 Lee, J., Macosko, C. W. \& Urry, D. W. Elastomeric polypentapeptides cross-linked into matrixes and fibers. Biomacromolecules 2, 170-179 (2001).

14 Zhang, S. G., Holmes, T. C., Dipersio, C. M., Hynes, R. O., Su, X. \& Rich, A. Self complementary oligopeptide matrices support mammalian-cell attachment. Biomaterials 16, 1385-1393 (1995).

15 Zhang, S. G., Lockshin, C., Herbert, A., Winter, E. \& Rich, A. Zuotin, a putative Z-DNA binding-protein in Saccharomyces-cerevisiae. EMBO J. 11, 3787-3796 (1992).

16 Holmes, T. C., de Lacalle, S., Su, X., Liu, G. S., Rich, A. \& Zhang, S. G. Extensive neurite outgrowth and active synapse formation on self-assembling peptide scaffolds. Proc. Natl. Acad. Sci. USA 97, 6728-6733 (2000).

17 Gelain, F., Horii, A. \& Zhang, S. G. Designer self-assembling peptide scaffolds for 3-D tissue cell cultures and regenerative medicine. Macro. Bioscience 7, 544-551 (2007)

18 Narmoneva, D. A., Vukmirovic, R., Davis, M. E., Kamm, R. D. \& Lee, R. T. Endothelia cells promote cardiac myocyte survival and spatial reorganization - implications for cardiac regeneration. Circulation 110, 962-968 (2004).

19 Puchtler, H. \& Sweat, F. Congo red as a stain for fluorescence microscopy of amyloid. J. Histochem. Cytochem. 13, 693-694 (1965).

20 Klunk, W. E., Pettegrew, J. W. \& Abraham, D. J. Quantitative-evaluation of congo red binding to amyloid-like proteins with a beta-pleated sheet conformation. J. Histochem. Cytochem. 37, 1273-1281 (1989). 